\title{
Conhecimentos e atitudes da equipe de enfermagem de uma maternidade na
}

\section{promoção da termorregulação neonatal}

\author{
Knowledge and attitudes of the nursing team of a maternity hospital in the promotion of neonatal
} thermoregulation

Conocimientos y actitudes del equipo de enfermería de una maternidad en la promoción de la termorregulación neonatal

\begin{abstract}
Resumo
Objetivo: analisar os conhecimentos e atitudes da equipe de enfermagem para promoção da termorregulação neonatal. Método: realizaram-se 11 entrevistas semiestruturadas, nos meses de novembro a dezembro de 2020, com membros da equipe de enfermagem da maternidade de um Hospital Universitário, posteriormente submetidas a análise temática e categorizadas em duas unidades temáticas intituladas: conhecimentos da equipe e atitudes da equipe. Resultados: percebeu-se uma lacuna de conhecimento entre profissionais técnicos e enfermeiros sobre o tema. Os participantes souberam elucidar corretamente as repercussões clínicas da termorregulação inadequada, e citaram os recursos materiais disponíveis para promovê-la, tais como berço aquecido e incubadora. Referente aos cuidados de enfermagem, principalmente o banho, percebeu-se que a equipe segue as recomendações da Organização Mundial da Saúde e do Ministério da Saúde, mas estas foram tardiamente implementadas na maternidade. Conclusão: a maioria das atitudes corroboram com as recomendações mais atuais para a promoção e manutenção da termorregulação do neonato.
\end{abstract}

Palavras-chave: Enfermagem neonatal; Hipotermia; Regulação da temperatura corporal; Recém-nascido; Alterações na temperatura corporal.

\begin{abstract}
Objective: to analyze the knowledge and attitudes of the nursing team to promote neonatal thermoregulation. Method: 11 semi-structured interviews were conducted in November/December 2020, with members of the maternity nursing team of a University Hospital, later submitted to thematic analysis and categorized into two thematic units entitled: team knowledge and team attitudes. Results: a knowledge gap was perceived between technical professionals and nurses on the subject. The participants were able to correctly elucidate the clinical repercussions of inadequate thermoregulation, and cited the material resources available to promote it, such as heated cradle and incubator. Regarding nursing care, especially bathing, it was noticed that the team follows the recommendations of the World Health Organization and Ministry of Health, but these were belatedly implemented in the maternity ward. Conclusion: most attitudes corroborate the most current recommendations for the promotion and maintenance of the thermoregulation of the neonate.
\end{abstract}

Keywords: Neonatal nursing; Hypothermia; Body temperature regulation; Newborn; Body temperature changes.

\section{Resumen}

Objetivo: analizar los conocimientos y actitudes del equipo de enfermería para promover la termorregulación neonatal. Método: En noviembre/diciembre de 2020 se realizaron 11 entrevistas semiestructuradas, con miembros del equipo de enfermería de maternidad de un Hospital Universitario, posteriormente sometidas a análisis temático y categorizadas en dos unidades temáticas tituladas: conocimiento del equipo y actitudes del equipo. Resultados: se percibió una brecha de conocimiento entre los profesionales técnicos y los enfermeros sobre el tema. Los participantes pudieron dilucidar correctamente las repercusiones clínicas de la termorregulación inadecuada, y citaron los recursos materiales disponibles para promoverla, como la cuna calentada y la incubadora. En cuanto a la atención de enfermería, especialmente el baño, se observó que el equipo sigue las recomendaciones de la Organización Mundial 
de la Salud y el Ministerio de Salud, pero estas fueron implementadas tardíamente en la sala de maternidad. Conclusión: la mayoría de las actitudes corroboran las recomendaciones más actuales para la promoción y mantenimiento de la termorregulación del neonato.

Palabras clave: Enfermería neonatal; Hipotermia; Regulación de la temperatura corporal; Recién nacido; Cambios en la temperatura corporal.

\section{Introdução}

No decorrer da gestação, a temperatura intrauterina é preservada por meio dos mecanismos maternos. Entretanto, após o nascimento, o neonato necessita adaptar-se ao meio externo relativamente mais frio e para isso produz calor através da elevação do seu metabolismo (Rolim et al., 2010). A termorregulação consiste na manutenção ideal da temperatura corporal através de controle fisiológico. Os valores de normalidade para os recém-nascidos (RN) estão entre $36,5^{\circ}$ e $37,5^{\circ} \mathrm{C}$. Fora dessa faixa, pode se considerar uma ameaça à homeostase, podendo acarretar distúrbios metabólicos e outras intercorrências, aumentando o risco de morbimortalidade neonatal (Martins et al., 2019).

O enfoque no controle da temperatura corporal do RN durante a assistência é primordial para sua sobrevida, visto que as alterações de temperatura do ambiente propiciam facilmente o resfriamento ou superaquecimento desses indivíduos (Rolim et al., 2010). A pele do RN é consideravelmente mais fina, condição que facilita a perda de fluidos e calor. Essa predisposição a perda, juntamente com a reduzida capacidade termorregulatória, pode impactar diretamente no êxito da adaptação cardiocirculatória e respiratória dos neonatos instantaneamente após o nascimento (Pugliesi et al., 2009; Suchy et al., 2018). Além disso, deve-se ter cautela em relação ao primeiro banho do RN, uma vez que consiste em um procedimento assistencial que pode prejudicar a adaptação extrauterina. Diante disso, verifica-se a importância de seguir as recomendações da Organização Mundial de Saúde (OMS) em relação ao primeiro banho, tais como adiá-lo em pelo menos 6 horas e manter contato pele-a-pele durante a primeira hora de vida para evitar hipotermia (de Lima et al., 2020; World Health Organization, 2018).

É infrequente a hipotermia ser fator causador de morte neonatal direta, mas está diretamente ligada a alterações metabólicas como hipoglicemia e hipóxia (de Freitas et al., 2018). Um estudo feito na Unidade de Terapia Intensiva Neonatal (UTIN) do Instituto de Medicina Integral Professor Fernando Figueira (IMIP) mostrou que os eventos adversos acometem 84\% dos RN internados, e $29 \%$ desse valor está representado por distúrbios de termorregulação, no qual 65,9\% são casos de hipotermia. ${ }^{8}$ Além da hipoglicemia, a hipotermia pode repercutir em risco ampliado de sepse, acidose metabólica, dificuldade respiratória e até morte (Howes \& Keir, 2018).

O RN pré-termo é ainda mais susceptível a inadequação da temperatura corporal, visto que uma série de mecanismos que auxiliam na promoção da termorregulação ainda não estão desenvolvidos de maneira satisfatória, tais como inabilidade para manter a flexão de extremidades, imaturidade acentuada do sistema nervoso para controle termorregulatório, desproporção entre superfície e massa corpórea, além da insuficiência de gordura marrom (de Freitas et al., 2018).

Após acompanhar, durante as vivências práticas do curso de enfermagem na maternidade de um Hospital Universitário, a rotina da equipe de enfermagem nos cuidados com o RN, surgiu o interesse em melhor compreender qual o nível de conhecimento desses profissionais acerca das ações para promover termorregulação dos neonatos, além de ter ciência de quais são suas condutas acerca desse tema, baseado nas recomendações da OMS.

Diante do exposto, elegeu-se a seguinte questão norteadora: quais os conhecimentos e atitudes da equipe de enfermagem de uma maternidade para promover a termorregulação dos recém nascidos? Neste sentido o objeto de estudo foi “os conhecimentos da equipe de enfermagem para a promoção da termorregulação de recém-nascidos".

O objetivo desse estudo foi analisar os conhecimentos e atitudes da equipe de enfermagem para promoção da termorregulação do RN, e compará-las com as recomendações da OMS e do Ministério da Saúde. 


\section{Metodologia}

Trata-se de um estudo qualitativo com enfoque no papel do profissional de enfermagem para promover a termorregulação dos recém-nascidos. A pesquisa qualitativa busca tratar a magnitude dos fenômenos, atenta a dimensão dos fatores (Minayo, 2017).

O estudo foi realizado em uma maternidade de um Hospital Universitário, na qual é composta pelo Centro Obstétrico (CO), Alojamento Conjunto (ALCON) e Unidade de Terapia Intensiva Neonatal (UTIN). A coleta de dados consistiu na realização de um total de 11 entrevistas semiestruturadas e audiogravadas, realizadas através da abordagem presencial em uma sala reservada na unidade, durante os meses de novembro e dezembro de 2020, feitas com membros da equipe de enfermagem da maternidade supracitada. A coleta foi encerrada quando atingiu a saturação teórica dos dados. Por saturação de dados, entende-se que ocorre quando o incremento não é mais necessário pois não há novas informações relevantes, de maneira que não altere o entendimento do estudo (Nascimento et al., 2018).

O roteiro de entrevista semiestruturado abordava questões relacionadas à definição de termorregulação, bem como quais são os seus achados e repercussões clínicas, e perguntas acerca das ações de promoção dos cuidados/orientações aos pais sobre o assunto. Além das entrevistas, os participantes preencheram um formulário de caracterização que abrangia questões referentes a categoria profissional, tempo de formação e de atuação na maternidade e se realizou curso de atualização ou especialização na área.

Os participantes foram selecionados através de amostragem por conveniência, dentre os quais foram incluídos enfermeiros e técnicos de enfermagem que estavam atuando na maternidade no período da coleta de dados. Foram excluídos aqueles profissionais de enfermagem de outros setores que se encontravam temporariamente na unidade ou que estavam de férias/licença. Não houve recusa ou desistência em participar da pesquisa.

Posteriormente todas as entrevistas foram transcritas e submetidas à análise temática, de acordo com os preceitos de Minayo, seguindo as três etapas recomendadas, quais sejam: pré-análise, exploração do material e tratamento dos resultados e interpretação (Minayo, 2010). Os dados foram analisados à luz das recomendações da OMS e do Ministério da Saúde. A fim de manter confidencialidade da identidade dos participantes, utilizou-se letras e números de acordo com a categoria profissional e ordem das entrevistas para descrevê-los nesse artigo, por exemplo: enfermeiro/entrevista 1 - E1 e técnico de enfermagem/entrevista 2 - TE2.

A pesquisa foi aprovada pelo Comitê de Ética em Pesquisa em Seres Humanos da Faculdade de Ciências da Saúde da Universidade de Brasília, número de parecer 4.036.601 e CAAE 29542920.0.0000.0030. Todas as etapas desse estudo respeitaram os preceitos da Resolução 466/2012 do Conselho Nacional de Saúde. Os entrevistados assinaram o Termo de Consentimento Livre e Esclarecido (TCLE) além do termo de autorização do uso de voz, ambos em duas vias, sendo uma cópia assinada entregue ao participante.

\section{Resultados}

Ao todo, foram realizadas 11 entrevistas, cinco com enfermeiros $(45,4 \%)$ e seis com técnicos de enfermagem $(54,6 \%)$. Estes possuem em média 15 anos de formação (mínimo cinco anos e máximo 28 anos) e atuavam, em média, há cinco anos na maternidade (mínimo seis meses e máximo 25 anos). Dos 11 participantes, cinco (45,4\%) afirmaram ter curso de atualização na área, dentre os quais três foi ofertado pelo hospital no qual atuam. Além disso, sete profissionais $(58,3 \%)$ afirmaram ter curso de especialização na área.

Após análise temática, as entrevistas foram divididas em dois temas: conhecimentos da equipe e atitudes da equipe. Posteriormente foram segregadas em subtemas de maior relevância, são eles: termos para definir termorregulação, 
identificação da temperatura adequada e repercussões clínicas da temperatura inadequada, inseridos no tema conhecimentos da equipe, e cuidados de enfermagem e orientações de enfermagem aos pais, inseridos no tema atitudes da equipe.

\subsection{Conhecimentos da equipe}

Essa unidade temática abrange os conhecimentos da equipe de enfermagem no que se refere as terminologias relacionadas à termorregulação, como é feita a identificação da temperatura adequada e as repercussões clínicas apresentadas pelos neonatos aos extremos de temperatura.

Quando questionados acerca da definição de termorregulação, os participantes demonstraram-se um pouco confusos com a palavra. Alguns relacionaram o termo diretamente à temperatura corporal, homeostase e parâmetros do RN, enquanto outros não souberam defini-lo. Percebeu-se uma lacuna de conhecimento entre profissionais técnicos e enfermeiros, na qual os enfermeiros souberam melhor elucidar.

Manter a hemodinâmica da temperatura do bebê, a homeostase na verdade, da temperatura do bebê desde a admissão até todo o acompanhamento dentro da UTI. (E1)

É quando ele consegue manter, por meios próprios, a regulação corporal térmica, sem afetar nenhuma estrutura, sem afetar a respiração. (E7)

Pra te falar a verdade eu nunca estudei isso a fundo. (TE9)

Temperatura... Parâmetros do RN. (TE4)

No que se refere a identificação da temperatura dos $\mathrm{RN}$, os entrevistados reportaram que fazem uso de termômetro e observam sinais que sugerem alteração, tais como coloração da pele, irritabilidade e choro.

Ao observar a olho nu, questão de coloração de pele, observo pela aferição de temperatura pelo termômetro digital [...]. (TE2)

O próprio bem estar mesmo, a gente vê que é um bebê que está tranquilo, não tá irritado. A gente fala muito do frio, mas o calor também irrita muito o bebê, aquele superaquecimento, aquele bebê que tá cheio de roupinha, [...] Fica irritadinho quando tá com calor e choroso também quando tá com frio. (E6)

Em relação as repercussões clínicas da temperatura inadequada, os profissionais alegaram como consequência da hipotermia neonatal a perda de peso, cansaço e perda da gordura marrom, sendo a última erroneamente associada a proteção do sistema imune.

Se ele [neonato] ficar frio ele pode perder peso, por conta da rápida assimilação do corpinho dele [...]. (TE3)

O bebê ele tem uma proteção fisiológica né que, ao nascer, à medida que ele tem essa perda de temperatura, ele começa a perder o que é conhecida como gordura marrom, então toda vez que esse bebé é exposto a uma temperatura menor que a temperatura corporal ele perde essa proteção, que é um protetor de sistema imunológico, então ele começa a perder isso aí. (E10)

Cansaço, a partir do momento que a gente aquece ele [neonato] a temperatura vai voltando ao normal, o bebê fica cansado, é o principal. (TE8)

Quanto a hipertermia neonatal, as repercussões clínicas mencionadas foram taquicardia, sudorese, taquipneia e desidratação. 


\section{[...] no caso da hipertermia geralmente dá taquicardia no bebê [...]. (TE3)}

Se tá com a temperatura aumentada a primeira coisa que a gente percebe é sudorese, taquicardia, pode aumentar a frequência respiratória devido à alta temperatura, e se for muito severo até uma desidratação. (E1)

Verifica-se que os participantes souberam responder aos questionamentos desta primeira categoria de maneira satisfatória, apesar da dificuldade apresentada no que se refere à alguns termos.

\subsection{Atitudes da equipe}

Essa unidade temática aborda as atitudes da equipe de enfermagem para a promoção da termorregulação do neonato, incluindo cuidados de enfermagem ofertados ao $\mathrm{RN}$ e as orientações fornecidas aos pais para não expor o bebê a temperaturas extremas.

Referente aos cuidados de enfermagem para promover a termorregulação dos RN, os participantes afirmaram que fazem uso da incubadora e berço aquecido para evitar a perda de calor, sendo que o manuseio do RN na incubadora é feito de forma breve, com apenas as portinholas abertas. Além disso, foi citado que é feito o uso de bolsas de água fria ou quente para tentar manter a homeotermia.

[...] quando a gente sabe que vai admitir um bebê a incubadora já está aquecida [...] durante a admissão tentar ser o mais breve possível e tentar realizar os procedimentos o máximo que a gente conseguir [...] com a porta fechada e só as portinholas abertas né [...]. (E1)

O berço aquecido que é o primeiro recurso que a gente tem [...]. (TE9)

Em alguns casos a gente pode usar bolsa de água fria ou bolsa de água quente. (TE3)

Em relação ao ambiente, foi apontado como cuidado de enfermagem desligar o ar condicionado.

\section{[...] desligar o ar-condicionado. (E11)}

Quanto aos cuidados com o banho do RN, houve divergência de falas acerca do adiamento do mesmo nas primeiras horas de vida. Alguns entrevistados alegaram que essa rotina foi implementada há cerca de um ano e outro afirmou que ocorreu há cerca de três anos. Também foi reportado que os bebês nascidos de mães HIV/HTLV positivas ou com presença de mecônio são banhados logo após o nascimento, e que a higienização dos demais neonatos consiste apenas em retirada do excesso de sangue e secreções com compressas secas. Houve um relato de que prematuros extremos não passam pelo procedimento de secagem, apenas são envoltos em saco plástico aquecido.

Na verdade, não dá mais o banho no CO, já tem quase 1 ano que isso foi abolido. Antigamente fazia um banho rápido e tudo, mas, quer dizer, deixa eu fazer uma ressalva: os bebês que nascem de mãe com HIV, aí sim, o protocolo é dar o banho, fora isso, não todos vem pra cá e a gente dá o banho com 12 horas de vida, 18, na verdade! Assim, nasceu, vamos supor, hoje à tarde, e aí só vai tomar banho amanhã de manhã. [...] Tira o excesso apenas, não é mais aquela limpeza completa como era antes, não vem tão limpinho mais não, a gente vê que ainda tem (vérnix) na cabeça, nas dobrinhas. (E6)

A gente dava banho, agora a gente não dá mais. Já faz tempo, tem mais de ano. E o banho dá em bebê de HIV e HTLV. E meconial dependendo dá. [...]. (TE8)

Assim, banho, desde que eu trabalho no CO que já não dava mais banho [...] 5 anos atrás, quando eu entrei aqui, a gente lavava a cabecinha e limpava com vaselina, e eu acho que deve ter mais ou menos uns 3 anos que foi cessado 
tudo isso. Agora assim, você seca o excesso de sangue e deixa, porque é a proteção deles, não pode mais deixar cheiroso. (TE9)

Só com pano seco e compressa seca. Não chega a dar banho e nem passar óleo. (E7)

[...] tirou, secou, a não ser que sejam prematuros, tem uns prematuros extremos você nem seca, coloca num saquinho aquecido né. (TE9)

Percebeu-se uma diferença de conduta na assistência ao parto normal e cesárea. Os profissionais referiram que o aquecimento dos campos ocorre apenas no parto cesárea, enquanto no parto normal esse aquecimento é dispensável, uma vez que é promovido através do contato pele-a-pele precoce com a mãe.

[...] as vezes aquece o campo pra colocar no neném [...] o aquecimento acontece principalmente na cesariana, mas se de parto normal não, porque a mãe promove essa termorregulação pós parto. (E7)

Na cesárea a gente também preconiza o contato pele a pele, só que é mais difícil pela posição da mãe, pela temperatura da sala que tem ar condicionado e pela condição que o bebê nasce, às vezes ele nasce com um pouquinho de líquido, tem que trazer pra aspirar. Já o parto normal quase sempre dá pra fazer [...]. (TE9)

Contato pele a pele, se o bebê estiver bem e a mãe também a gente faz esse contato imediato, [...], mas a gente sempre tá em cima na questão do contato pele a pele [...]. (E7)

Quando questionados sobre as orientações de enfermagem aos pais para que promovam a termorregulação de seus filhos, os participantes disseram que os instruem acerca de quais condutas devem tomar a fim de evitar hipotermia ou superaquecimento. Entre as orientações estão a troca de roupa e percepção da sensação térmica.

Eu particularmente nunca dei orientação sobre superaquecimento. A gente orienta sempre em relação a manter na temperatura ideal, se tiver muito calor desafogar um pouco das roupinhas, colocar uma roupinha mais leve, mais neutra e se tiver muito frio aquecer o bebê direitinho com manta, com roupinha, com meia e luva. (E5)

Sim, a gente orienta que o bebê no primeiro momento sente muito frio, mas depois a temperatura é de acordo com a sua também, se você tá com frio seu bebê também vai sentir frio, se não, se tá mais quente você tem que colocar roupas mais leves. (TE9)

Os participantes souberam apontar quais são os cuidados de enfermagem para promoção da termorregulação noenatal realizados na maternidade desse estudo de maneira satisfatória, porém percebeu-se que os profissionais técnicos não compreendiam por completo a razão pelas quais realizam essas condutas, apenas as fazem de maneira mecânica.

\section{Discussão}

A termorregulação consiste em uma função fisiológica que permite o controle e manutenção de um ambiente corporal neutro (Lima et al., 2020). Percebeu-se, ao questionar os participantes sobre o que entendem por termorregulação do RN, uma discordância de respostas entre profissionais de nível superior e técnico, nos quais os enfermeiros souberam elucidar o conceito de acordo com a literatura, relacionando termorregulação à fisiologia, enquanto os técnicos de enfermagem associaram a parâmetros do RN ou não souberam responder. Mesmo que grande parte dos participantes desse estudo tenham realizado cursos de especialização/atualização na área, essa diferença pode ser oriunda de uma formação técnica inicial na qual a prática é mais enfatizada do que a teoria. Um estudo demostrou que os profissionais técnicos que tiveram a oportunidade de fazer curso de graduação se sentem mais satisfeitos, uma vez que entendem o porquê de realizar as atividades, e não somente as executarem sem completo entendimento (LF, 2020). 
Por interagir com o ambiente, a temperatura do RN pode apresentar constante mudança, portanto exige avaliação frequente e contínua pelos profissionais. O termômetro digital é o equipamento de preferência para avaliar a temperatura central. Entre as manifestações clínicas da hipotermia está a vasoconstrição, que pode manifestar-se através de sinais como pele com coloração vermelho brilhante devido a falha na dissociação da oxihemoglobina (Brasil, 2011), enquanto na hipertermia, durante o ganho de calor por convecção, o RN pode ficar agitado, irritado e adquirir a posição distendida, na tentativa de aumentar a transferência de calor (Aquino et al., 2018). Diante disso, constata-se que os participantes desse estudo têm conhecimento quanto às manifestações clínicas ocorridas durante as oscilações de temperatura do RN, assim como fazem o correto uso dos dispositivos para identificar a temperatura adequada do RN.

Quando exposto ao frio, o RN promove vasoconstrição pulmonar e periférica, além de elevar seu metabolismo basal. Como consequência, ocorre o aumento do consumo de oxigênio nos tecidos, fator que o predispõe à hipóxia e acidose metabólica devido ao aumento do metabolismo anaeróbio. Como forma de compensar esse aumento e melhor oxigenar os tecidos, o RN eleva sua frequência cardíaca e respiratória. Caso as alterações fisiológicas não sejam normalizadas, o elevado consumo de oxigênio nos tecidos pode acarretar uma diminuição do suprimento às células cardíacas, resultando em bradicardia (R. O. de Lima et al., 2020; Rolim et al., 2010).

Além dessas alterações, o RN exposto ao frio inicia um processo de lipólise de gordura marrom, através da liberação de noradrenalina e hormônios tireoidianos, conhecido como termogênese química, que consiste no principal mecanismo de produção de calor no RN. A perda dessa gordura está associada a perda de peso, uma vez que é considerada uma reserva calórica peculiar (Brasil, 2017; Brasil, 2011), corroborando com a fala dos entrevistados desse estudo acerca das repercussões clínicas da hipotermia. Em contrapartida, erroneamente um dos participantes do presente estudo associou a perda da gordura marrom à diminuição da imunidade do RN, evidenciando a incompreensão acerca de sua fisiologia e a necessidade de aperfeiçoamento de seus conhecimentos acerca dessa temática.

Referente à hipertermia, o Ministério da Saúde aponta como possíveis causas a desidratação e condições ambientais favoráveis ao superaquecimento, sendo a última causadora de hipoatividade no RN. Atribui-se perda acentuada de peso como uma das consequências da desidratação. A hipertermia pode ser muito prejudicial ao tecido cerebral, uma vez que o cérebro é bastante sensível a alterações térmicas. Apesar das glândulas dos RN ter capacidade de resposta reduzida ao nascer, a pele do RN é muito fina e permite intensas trocas de calor com o ambiente. Esse mecanismo permite manter a homeostase mediante superaquecimento através da sudorese (Brasil, 2017; Brasil , 2011).

Os sinais vitais do RN indicam estabilidade orgânica, portanto qualquer mudança pode apontar estresse e estafa, provocando desequilíbrio (R. O. de Lima et al., 2020). Diante disso, pode se afirmar que a má manutenção da homeotermia predispõe o neonato a alteração dos parâmetros vitais, insuficiência respiratória, sudorese, desidratação, perda de peso e cansaço, corroborando com o apontamento dos participantes desse estudo acerca das repercussões clínicas da temperatura inadequada. Além dessas alterações, o Ministério da Saúde aponta outras manifestações clínicas da hipotermia, tais como bradicardia, hipotensão, hipóxia, hipoglicemia e acidose (Brasil, 2011), nas quais não foram mencionadas pelos entrevistados.

Para evitar as alterações clínicas, a unidade deve dispor de recursos materiais para auxiliar nesse processo. Entre os recursos disponíveis na maternidade do presente estudo para promover a termorregulação dos $\mathrm{RN}$ foram citados a incubadora e o berço aquecido. Esses instrumentos também são preconizados pelo Ministério da Saúde para se obter o ambiente de termoneutralidade em unidades neonatais. Dentro da incubadora o ar é aquecido por convecção, portanto, toda vez que as portinholas são abertas para manuseio do RN a temperatura se altera, evidenciando a necessidade de abri-las o mínimo possível (Brasil, 2011). O berço aquecido é bastante utilizado para recepção do RN e realização de procedimentos, e sua temperatura é mantida através de calor radiante, porém seu uso de maneira inadequada pode gerar hipertermia (L. da S. Lima et al., 2020; Brasil, 2011). 
Além dos recursos mencionados acima, um participante apontou que faz uso de bolsas de água fria ou quente como cuidado de enfermagem para promover um ambiente corporal neutro nos RN. É importante ressaltar que a pele fina do neonato o predispõe a maior risco de lesões, dessa forma, bolsas térmicas podem ser potenciais causadores de queimaduras. Apesar de rara, a queimadura no RN pode ocorrer em consequência da sensibilidade cutânea, sendo que estas podem ser de etiologia física ou química. Entre os fatores de risco dessas lesões estão: oxímetro de pulso, lâmpadas de aquecimento infravermelhas, agentes químicos para antissepsia e aplicação de calor local (Saavedra et al., 2018).

No que se refere às precauções com o ambiente para promover a termorregulação, desligar o ar condicionado foi um dos cuidados de enfermagem citados pelos entrevistados. É importante atentar-se ao ambiente desde o parto, tendo em vista a diferença de temperatura do ambiente intra e extra uterino, a fim de reduzir o impacto dessa diferença para o neonato (L. da S. Lima et al., 2020). A transição de temperatura entre esses ambientes propícia perda de calor por evaporação e convecção, reduzindo a temperatura do $\mathrm{RN}$ cerca de $0,3^{\circ}$ por minuto (Brasil, 2011).

Quanto aos cuidados com o primeiro banho do neonato, algumas falas foram divergentes acerca do adiamento nas primeiras horas de vida, visto que houveram relatos de que a implementação dessa rotina ocorreu há cerca de um ano e outro afirmou que ocorreu há cerca de três anos. Desde 2013 a OMS recomenda alguns cuidados com os RN, entre eles está o adiamento do primeiro banho em pelo menos 24 horas após o nascimento e caso não seja possível, devido a questões culturais, adiar em pelo menos 6 horas (World Health Organization, 2018). Embora as falas dos participantes sejam controversas, a implementação dessa rotina de adiamento deveria ter sido realizada antes do prazo citado, evidenciando a lenta adesão aos protocolos das autoridades de saúde.

A adaptação do RN ao meio extrauterino pode sofrer intervenções causadas pelo banho, uma vez que este pode promover alteração dos sinais vitais, da proteção térmica e da pele. Apesar de imatura, a pele do neonato possui funções de proteção contra agentes nocivos através do vérnix caseoso, que dispõe de propriedade antimicrobiana, hidratante e de redução da descamação (R. O. de Lima et al., 2020). Perante o exposto, observa-se que a conduta dos profissionais desse estudo em retirar apenas o excesso de sangue e secreções com compressas secas, sem limpar o vérnix, é concórdia com o preconizado pela literatura.

Em relação ao banho dos bebês nascidos de mães HIV/HTLV positivas, os profissionais desse estudo afirmaram que o mesmo é realizado logo após o nascimento. Essa conduta corrobora com as diretrizes terapêuticas preconizadas pelo Ministério da Saúde, que recomendam realizar o banho na sala de parto, de preferência com fonte de água corrente, e a limpeza delicada de todo o sangue e secreções visíveis com compressas macias, com cuidado, a fim de evitar lesões na pele da criança e uma possível contaminação (Brasil, 2019).

Ainda sobre os cuidados pós parto, o uso de saco plástico foi apontado como barreira contra perda de calor em prematuros menores que 28 semanas. O RN é colocado dentro do mesmo até a altura do pescoço, sem passar por secagem, a fim de diminuir o risco de hipotermia (Brasil, 2011). Diante disso, evidencia-se mais uma intervenção realizada pelos profissionais entrevistados que corrobora com as recomendações da literatura.

As falas dos participantes desse estudo revelam que os mesmos possuem condutas diferentes na assistência ao parto normal e cesárea no que se refere aos cuidados para promover a termorregulação. Os campos utilizados para envolver no RN são aquecidos apenas no parto cesárea, com justificativa de que no parto normal o aquecimento é substituído pelo contato pelea-pele precoce com a mãe. Essa diferença é divergente do que é recomendado pelo Ministério da Saúde em que preconiza que os cuidados de rotina, ao nascimento, para prevenir hipotermia, incluem pré-aquecer os campos em fonte de calor radiante e recepcionar o $\mathrm{RN}$ em campos aquecidos, além do contato pele-a-pele precoce, se possível, não distinguindo a conduta entre parto normal e cesárea (Brasil, 2011). 
Referente a orientação aos pais, para que possam promover a termorregulação de seus filhos, os participantes demonstraram instrui-los corretamente, de forma de abrange a prevenção de hipo e hipertermia. Entre as atribuições da equipe multiprofissional que atua em ALCON está a realização de atividades de educação em saúde com o objetivo de ampliar o cuidado com os neonatos (Brasil, 2016).

Como limitação do estudo, aponta-se que foi realizado apenas com um grupo de participantes que atuam na maternidade de uma única instituição, portanto os resultados não podem ser generalizados.

\section{Conclusão}

Foi possível concluir, através da análise dos conhecimentos e cuidados realizados pelos participantes desse estudo para a promoção da termorregulação do RN, que a maioria das atitudes corroboram com as recomendações da OMS e do Ministério da Saúde, apesar de algumas terem sido tardiamente implementadas ou em desacordo com o que é preconizado pela literatura atual. Além disso, percebeu-se que a lacuna de conhecimento sobre a temática por parte dos profissionais de nível técnico, o que pode acarretar alguns prejuízos aos neonatos devido à condutas equivocadas, evidenciando a importância da educação continuada e atualização constante para melhor ofertar os cuidados de enfermagem, mitigando as iatrogenias.

A avaliação do conhecimento dos profissionais de enfermagem coopera com a segurança do paciente, uma vez que levantando os pontos de fragilidade, a equipe pode se reajustar e autopromover um processo de melhoria contínua, acarretando uma assistência de qualidade. Espera-se que essa pesquisa contribua com esse processo.

Nota-se que a literatura possui poucos estudos acerca dos conhecimentos dos profissionais na promoção da termorregulação. Portanto, sugere-se estudos nas mais variadas maternidades e inserir os gestores das maternidades nas pesquisas, a fim de compreender quais ações realizadas por eles contribuem para ampliar os conhecimentos da equipe.

\section{Referências}

Aquino, W., Lopes, M., Silva, V., Fróes, N., Menezes, A., Almeida, A., \& Sobreira, B. (2018). Acurácia das características definidoras do diagnóstico de enfermagem hipertermia em recém-nascidos. 71(2), 380-386.

Brasil. Ministério da Saúde. (2011). Atenção à saúde do recém-nascido: guia para os profissionais de saúde. Normas e Manuais Técnicas.

Brasil. Ministério da Saúde. (2017). Atenção humanizada ao recém-nascido: Método Canguru. Manual técnico.

Brasil. Ministério da Saúde. (2016). Portaria $n^{\circ}$ 2.068, de 21 de outubro de 2016. Diário Oficial Da União, ISS 1677-7, 1-2.

Brasil. Ministério da Saúde. (2019). Protocolo clinico e diretrizes terapeuticas para prevenção da transmissão vertical de HIV, Sífilis e Hepatites virais. Protocolo clínico.

de Freitas, P., Munhoz, M. M. B., Costa, P., \& Kimura, A. F. (2018). Efeito de duas técnicas de banho de imersão na temperatura axilar de recém-nascidos pré-termos: Estudo piloto. Texto e Contexto Enfermagem, 27(1), 1-8.

Howes, A., \& Keir, A. (2018). Quality improvement effort to reduce hypothermia among high-risk infants on a mother-infant unit. Acta Paediatrica, International Journal of Paediatrics, 107(10), 1837.

LF, F. mt e M. (2020). Integração ensino-serviço na formação técnica de enfermagem. Rev. Eletr. Enferm,2020, 1-7.

Lima, R. O. de, Estevam, L. D., Leite, F. M. C., Almeida, M. V. S., Nascimento, L., Amorim, M. H. C., \& Bringuente, M. E. de O. (2020). Intervenção de enfermagem-primeiro banho do recém-nascido: estudo randomizado sobre o comportamento neonatal. Acta Paulista de Enfermagem, 33(12), 128-139.

Lima, L. da S., Reis, E. A. F., da Silva, E. M., \& Moura, J. P. G. (2020). Nursing care in the thermo-regulation of preterm newborns: An integrative review. Cogitare Enfermagem, 25, 1-12.

Martins, L. A., Silveira, S. P. X. da, Avila, I. M. F. T., Moraes, J. A. S. de, Santos, D. S. S. dos, Whitaker, M. C. O., \& Camargo, C. L. de. (2019). Implantação do protocolo de termorregulação para recém-nascido em procedimentos cirúrgicos TT. Rev. Gaúch. Enferm, 40(spe), e20180218-e20180218.

Minayo, M. C. de S. (2017). Sampling and saturation in qualitative research: consensuses and controversies. Revista Pesquisa Qualitativa, 5(7), 1-12.

Minayo, M. C. de S. (2010). Minayo MCS. O desafio do conhecimento. Pesquisa qualitativa em saúde. $9^{a}$ edição revista e aprimorada. São Paulo: Hucitec; 2006. 406 p. Hucitec, $1087-1088$. 
Research, Society and Development, v. 10, n. 10, e593101019110, 2021

(CC BY 4.0) | ISSN 2525-3409 | DOI: http://dx.doi.org/10.33448/rsd-v10i10.19110

Nascimento, L. de C. N., Souza, T. V. de, Oliveira, I. C. dos S., Moraes, J. R. M. M. de, Aguiar, R. C. B. de, \& Silva, L. F. da. (2018). Saturação teórica em pesquisa qualitativa: relato de experiência na entrevista com escolares. Rev Bras Enferm [Internet], 71(1), $243-251$.

Pugliesi, V. E. M., Deutsch, A. D., Freitas, M. de, Dornaus, M. F. P. S., \& Rebello, C. M. (2009). Efeitos do banho logo após o nascimento sobre as adaptações térmica e cardiorrespiratória do recém-nascido a termo. Revista Paulista de Pediatria, 27(4), 410-415.

Rolim, K. M. C., Araújo, A. F. P. C., Campos, N. M. M., Lopes, S. M. B., Gurgel, E. de P. P., \& Campos, A. do C. S. (2010). Cuidado quanto à termorregulação do recém-nascido prematuro: o olhar da enfermeira. Rev. RENE, 11(2), 44-52.

Saavedra, P. A. E., Soares, L., Nascimento, S. B., Oliveira, J. V. L. de, Areda, C. A., Cunha, K. B. da, Adorno, J., \& Galato, D. (2018). Os rastreadores de eventos adversos a medicamentos se aplicam a pacientes hospitalizados por queimaduras? Rev. Bras. Queimaduras, $17(1), 20-27$.

Suchy, C., Morton, C., Ramos, R. R., Ehrgott, A., Quental, M. M., Burridge, A., \& Rutledge, D. N. (2018). Does Changing Newborn Bath Procedure Alter Newborn Temperatures and Exclusive Breastfeeding? Neonatal Network : NN, 37(1), 4-10.

Ventura, C. M. U., Alves, J. G. B., \& Meneses, J. do A. (2012). Eventos adversos em Unidade de Terapia Intensiva Neonatal. Revista Brasileira de Enfermagem, 65(1), 49-55.

World Health Organization. (2018). Intrapartum care for a positive childbirth experience. Protocolo. 